\title{
Neuropsychiatric Symptoms in Inborn Errors of Metabolism: Incorporation of Genomic and Metabolomic Analysis into Therapeutics and Prevention
}

\author{
Lisa Pan · Jerry Vockley
}

Published online: 8 January 2013

(c) Springer Science + Business Media New York 2013

\begin{abstract}
Inborn errors of metabolism may present as a spectrum ranging from neonatal lethality to non-specific symptoms. Neuropsychiatric manifestations have been identified in three groups: those presenting as emergencies, those with chronic fluctuating symptoms, and those associated with mental retardation. Milder central nervous system specific inborn errors of metabolism may also present later in life with isolated psychiatric symptoms. Inborn errors of metabolism presenting with neuropsychiatric symptoms are described with illustrative case examples. Metabolomic and genomic approaches to identification and treatment are described.
\end{abstract}

Keywords Inborn error of metabolism - Neuropsychiatric . CNS-specific · Genomics · Metabolomics · Psychosis · Depression $\cdot$ Suicide

\section{Introduction}

Inborn errors of metabolism (IEMs) are traditionally defined as defects in a single gene leading to a physiologically relevant decrease in the function of an enzyme. They can present with a spectrum of symptoms ranging from neonatal

\section{Pan}

School of Medicine, Western Psychiatric Institute and Clinic, University of Pittsburgh, 100 N. Bellefield Ave., Rm. 320, Pittsburgh, PA 15213, USA

e-mail: thomasla@upmc.edu

\section{J. Vockley $(\bowtie)$}

School of Medicine, Graduate School of Public Health, University of Pittsburgh, Children's Hospital of Pittsburgh of UPMC, 401 Penn Avenue, Pittsburgh, PA 15224, USA e-mail: gerard.vockley@chp.edu lethality due to accumulation of an intoxicating compound to onset of non-specific symptoms later in life. Delayed diagnosis in the latter situation can lead to significant morbidity and mortality. Neuropsychiatric manifestations in IEMs have historically been grouped into three classifications, those presenting as emergencies (delirium, confusion), those with chronic, fluctuating psychiatric symptoms, and those presenting in the setting of mild mental retardation [1]. Early identification when psychiatric symptoms are present is crucial to prevent permanent neurologic damage in these settings (Table 1).There is, however, a fourth group of milder central nervous system (CNS) specific IEMs presenting later in life with isolated neuropsychiatric symptoms, currently not identified by most diagnostic paradigms. In addition, psychiatric symptoms, neurocognitive difficulties, and learning disability are well documented in patients with known IEMs, even in the setting of appropriate treatment. Examples include phenylketonuria [2, 3], maple sugar urine disease [4, 5], Wilson's disease [6], the porphyrias [7], Wolfram's syndrome [8], and homocystinuria [9, 10]. Imbalances in dopamine and serotonin pathways related to mood, anxiety, learning and cognition (for review see [11, 12]) may contribute to the development of these symptoms in patients with IEMs.

Consideration of single gene defects leading to IEMs with psychiatric manifestations almost certainly underestimates the scope of the problem. The human genome consists of 3 billion nucleotide base pairs encoding 25,000 genes, and more than 4,000 specific genetic disorders affecting $1 \%$ of all live births. Nonetheless, single gene effects do not explain all genetic disease, as genotypephenotype correlates are imperfect [13]. Dysfunction at multiple gene loci can also result in disease, and disease modifying factors complicate our understanding of IEMs [14••]. For example, multiple partial enzyme deficiencies 
Table 1 Known inborn metabolic disorders that may present with psychiatric symptoms

\begin{tabular}{|c|c|c|c|}
\hline Disorder & Symptoms & Signs & Psychiatric presentation \\
\hline \multicolumn{4}{|l|}{ Intoxication syndromes } \\
\hline Urea cycle disorders & Liver dysfunction, emesis & NH3, AA, orotic Acid & Confusion \\
\hline $\begin{array}{l}\text { Homocysteine } \\
\text { remethylation defects }\end{array}$ & Stroke, seizures, myelopathy & Homocystinuria & $\begin{array}{l}\text { Behavioral changes } \\
\text { Psychosis }\end{array}$ \\
\hline $\begin{array}{l}\text { Cystathionine- } \beta \text { synthase } \\
\text { deficiency }\end{array}$ & $\begin{array}{l}\text { Seizure, osteoporosis, } \\
\text { thromboembolism, eye d/o }\end{array}$ & Homocystinuria & Delirium \\
\hline $\begin{array}{l}\text { Acute intermittent } \\
\text { porphyrias }\end{array}$ & Abdominal pain, emesis, neuropathy & Dark urine, plasma/urine porphyrins & \\
\hline Phenylketonuria & Seizure, developmental delay & Hyperphenylalanemia & \\
\hline \multicolumn{4}{|c|}{ Lipid storage/oligosaccharidoses/mucopolysaccaridoses } \\
\hline Niemann-pick C & Vertical opthalmoplegia & Bone marrow foam cells & Psychosis \\
\hline GM2 gangliosidosis & Ataxia, dystonia, polyneuropathy & Enzyme testing, CSF GM2 & Delusions \\
\hline $\begin{array}{l}\text { Metachromatic } \\
\text { leukodystrophy }\end{array}$ & $\begin{array}{l}\text { Ataxia, dystonia, demyelinating } \\
\text { polyneuropathy }\end{array}$ & $\begin{array}{l}\text { Leukocyte arylsulfatase A; MRI symmetrical } \\
\text { white matter changes }\end{array}$ & $\begin{array}{l}\text { Disorganization } \\
\text { Catatonia }\end{array}$ \\
\hline Adrenoleukodystrophy & $\begin{array}{l}\text { Paraparesis, adrenal insufficiency, } \\
\text { neuropathy }\end{array}$ & MRI may show leukoencephalopathy & $\begin{array}{l}\text { Cognitive decline } \\
\text { (mimics schizophrenia) }\end{array}$ \\
\hline $\begin{array}{l}\text { Cerebrotendinous } \\
\text { xanthomatosis }\end{array}$ & $\begin{array}{l}\text { Variable neurological symptoms, } \\
\text { osteoporosis, xanthomas, }\end{array}$ & $\begin{array}{l}\text { Cerebellar atrophy, } \\
\text { low Vit. D }\end{array}$ & \\
\hline$\alpha$-Mannosidosis & Ataxia & Leukoencephalopathy & \\
\hline Ceroid lipofuscinosis & $\begin{array}{l}\text { Seizures, retinitis } \\
\text { pigmentosa }\end{array}$ & None & \\
\hline $\begin{array}{l}\alpha \text {-Methyl-acyl-CoA } \\
\text { racemase deficiency }\end{array}$ & $\begin{array}{l}\text { Encephalopathy, seizure, neuropathy, } \\
\text { retinopathy }\end{array}$ & Elevated serum pristanic acid & \\
\hline \multicolumn{4}{|l|}{ Metal storage disorders } \\
\hline Wilson's disease & Liver disease, movement disorder & Low serum ceruloplasm, copper & Impulsivity \\
\hline Aceruloplasminemia & $\begin{array}{l}\text { Retinal degeneration, Diabetes } \\
\text { mellitus, Neurological changes }\end{array}$ & $\begin{array}{l}\text { Low serum ceruloplasm, iron, copper, high } \\
\text { serum ferritin, Brain MRI iron deposits }\end{array}$ & $\begin{array}{l}\text { Irritability } \\
\text { Psychosis }\end{array}$ \\
\hline Neuroferritinopathy & Extrapyramidal symptoms & Low plasma ferritin & \\
\hline $\begin{array}{l}\text { PANK2 (pantothenate } \\
\text { kinase 2) }\end{array}$ & Neurodegeneration & MRI iron deposits in the basal ganglia & \\
\hline \multicolumn{4}{|c|}{ Central nervous system specific disorders } \\
\hline $\begin{array}{l}\text { Disorders of } \\
\text { neurotransmitter } \\
\text { metabolism }\end{array}$ & Neuropsychiatric & CSF HVA, 5-HIAA, neopterin, biopterin & $\begin{array}{l}\text { Depression } \\
\text { Suicide } \\
\text { Variable }\end{array}$ \\
\hline $\begin{array}{l}\text { Cerebral folate } \\
\text { deficiency }\end{array}$ & Neuropsychiatric & CSF 5MTHF & \\
\hline
\end{tabular}

Adapted from [35]

have been shown to lead to clinically relevant dysfunction in muscle energy metabolism, with evidence of an additive effect of heterozygous mutations in multiple genes in functionally related pathways [15]. Heterozygosity for multiple defects in a metabolic pathway or functionally related pathways has been termed "synergistic heterozygosity" [15], a concept with potential implications in the diagnosis, treatment, and understanding of CNS specific IEMs leading to psychiatric symptoms. Physiologically, this reflects reduction of flux through pathways, or a dominant negative model in which one protein with an unstable mutation disrupts the structure of a complex needed for appropriate function of the pathway. Such epistasis or multigenic inheritance can just as easily lead to a CNS specific disease phenotype. Relevant examples include dihydrofolate reductase in the folate metabolism pathway [16], and guanosine triphosphate (GTP) cyclohydrolase and associated tetrahydrobiopterin (BH4) deficiencies in the serotonin, dopamine, and nitrous oxide pathways $[17,18,19 \bullet]$.

Disorders of metabolism in psychiatric disease, particularly aberration of flux through metabolic pathways rather than complete deficiencies, may be far more common than we are aware. A study of 34 adult psychiatric patients with 
severe major depressive disorder revealed that $21 \%$ had previously unrecognized reduced folate levels $(<150 \mathrm{ng} /$ $\mathrm{ml}$ ), which correlated with lower cerebrospinal fluid (CSF) 5-HIAA [20]. Of 673 children with elevated phenylalanine screened for biopterin synthesis defects with HPLC of urine pterins and a biopterin load test, one child with GTP cyclohydrolase I deficiency, 36 children with dihydrobiopterin synthetase deficiency (DHBS), and 14 children with dihydropteridine reductase (DHPR) were identified [21]. In this sample, $7.5 \%$ were found to have an IEM.

Over the past two decades, improvements in the diagnosis of IEMs have been driven by the development of new technologies to identify groups of metabolites indicative of a specific diagnosis. Gas chromatography of urine organic acids revolutionized the identification of the organic acidemias, and tandem mass spectrometry (MS/ MS) analysis of the blood has extended the range of disorders that can readily be identified in clinical practice, with the advantage of requiring very small sample volumes. Linking both technologies to a variety of front-end columns for sample fractionation dramatically expands their ability and can lead to the identification of literally thousands of physiologically and clinically relevant compounds in body fluids. Metabolomics is a relatively new and rapidly evolving field that focuses on the relationship of metabolites to physiologic and pathophysiologic processes, but the relationship of metabolic balance to deficiencies to mental health disorders is understudied. One metabolomic study of nine geriatric patients with depression suggested that the depressed state may be associated with alterations in the metabolism of lipids and neurotransmitters, particularly GABA, but cause and effect could not be determined [22•]. Blood biopterin level and inflammatory compounds have been correlated with depression in older adults as well (for review see [23]). Metabolomics techniques have also been utilized to study Huntingdon's disease, Parkinson's disease, and schizophrenia (for review see [24*0]). In addition to deficiency of specific cofactors having the potential to adversely affect monoamine synthesis, disorders of mitochondria have also been implicated in psychiatric disorders (for review see [25]). Mitochondria are the main intracellular producers of oxidants, and mitochondrial disorders may impact the metabolism of monoamines and lead to an increased risk of depression. Mitochondria are also involved directly in monoamine synthesis via monoamine oxidases (MAO) in the mitochondrial membrane. Finally, individual structural genetic variants have been identified in genomics studies both in studies of schizophrenia [26] and in large scale studies seeking drug targets [27]. These structural variants are abundant and may contribute to deleterious effects similar to the cases described below.

\section{Illustrative Clinical Cases}

Guanosine triphosphate-cyclohydrolase (GTPCH) (Fig. 1) deficiency is an autosomal-recessive genetic disorder associated with neurologic abnormalities. Type I includes hyperphenylalaninemia due to deficiency of BH4. In milder variants, defective monoamine production is prominent because of $\mathrm{BH} 4$ dependent tyrosine and tryptophan hydroxylases. Biopterin is a cofactor for three enzymes: the conversion of phenylalanine-4-hydroxylase (to phenylalanine), tyrosine-3-hydroxylase (to catecholamines) and tryptophan-5-hydroxylase (to serotonin). Nearly 200 different mutant alleles in GTPCH have been identified. Treatment with sapropterin (Kuvan) and/or the deficient monoamine may be successful. We have recently reported the treatment of a 19-year-old patient with treatmentrefractory suicidal ideation and severe major depressive disorder who had a CSF metabolite profile consistent with GTPCH deficiency. He had an IQ in the gifted range and other neurologic abnormalities were absent. He presented at 14 years of age with suicide attempt by overdose, and by age 15 years, he overdosed again requiring treatment in the pediatric intensive care unit. Despite treatment with serotonin-norepinephrine reuptake inhibitors (SNRIs), riluzole, antipsychotics, mood stabilizers and electroconvulsive therapy (ECT) (Fig. 1), the patient did not improve. Serotonin reuptake inhibitors (SSRIs) resulted in worsening. At age 17 years, after 32 treatments of ECT, he remitted for only 1 week followed by immediate onset of suicidal intent and an aborted suicide attempt. The patient refused further ECT due to non-response. He demonstrated significant suicidality during a 49-day hospitalization. With maximal dose polypharmacy, the patient experienced neither improvement nor side effects. Neurologic examination remained normal throughout.

Metabolic testing revealed normal cytochrome $\mathrm{P} 450$ (3A4, 2D6 and C19) metabolism and homozygous long allele of the serotonin transporter promoter gene (SLC64A) locus. Brain MRI was normal. CSF showed undetectable neopterin, biopterin $10 \mathrm{nM} / 1$ (12-30), 5-hydroxyindoleacetic acid (5HIAA) $38 \mathrm{nM} / 1$ (67-140) and homovanillic acid (HVA) $116 \mathrm{nM} / 1$ (145-324), indicative of GTPCH deficiency, and thus a defect in the pterin biosynthetic pathway. Blood phenylalanine level was $6 \mu \mathrm{mol} / \mathrm{dl}(\mathrm{nl}<12)$ and sequencing of the GTPCH gene showed no pathogenic mutation. CSF 5MTHF was normal (levels 93 and 98, range 40-120).

The patient was started on sapropterin $800 \mathrm{mg}(10 \mathrm{mg} / \mathrm{kg})$ supplementation. Beginning treatment day 6 , he reported euphoric mood, then euthymia; on day 17 , hyperactivity, euphoria and normal sleep. By day 24, his mood fluctuated markedly with gradually decreasing frequency. The patient reported mood incongruent impulses and strong feelings of love and emotion. By day 31, the effect plateaued. 
TREATMENT

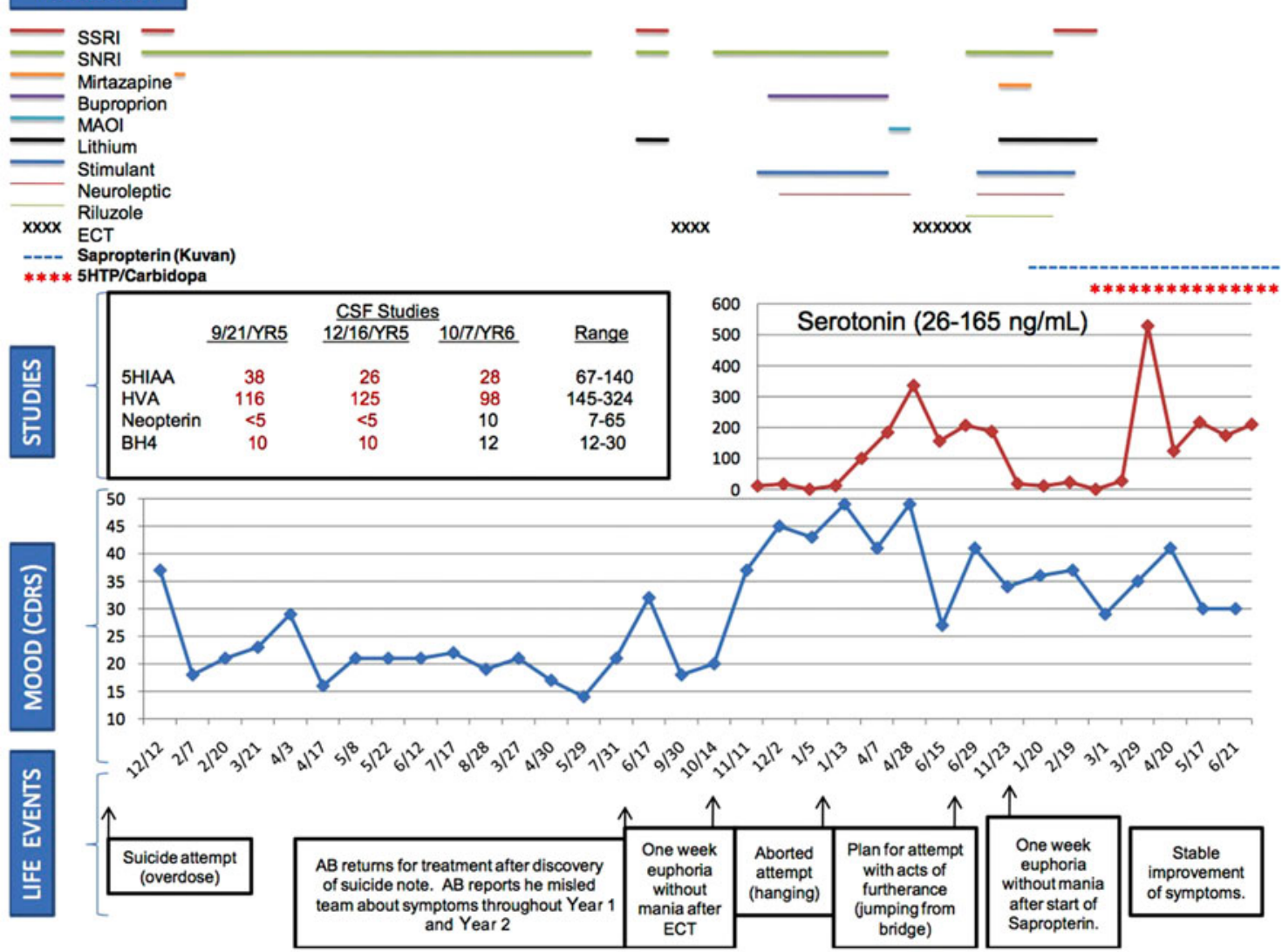

Fig. 1 Course and treatment of treatment refractory depression in a patient with a new variant of GTP-cyclohydrolase deficiency [19•]. CDRS children's depression rating scale, SSRI selective serotonin reuptake inhibitor, $S N R I$ serotonin-norepinephrine reuptake inhibitor,

Sapropterin was increased to $800 \mathrm{mg}$ orally twice daily $(20 \mathrm{mg} / \mathrm{kg})$. On day 40, the CSF neurotransmitter pattern revealed little change (Fig. 1). He began to have episodes of shaking, insomnia and word-finding difficulty. Sapropterin was reduced to $800 \mathrm{mg}$ orally, in the morning and $400 \mathrm{mg}$ orally, at bed time. After 2.5 months, the patient reported stable improvement, but mood remained low. He received 5-hydroxytryptophan (5-HTP) supplementation with carbidopa to block peripheral effects of serotonin and increase conversion to serotonin in the CNS. Sapropterin was stabilized at $700 \mathrm{mg}$ orally twice daily and carbidopa $25 \mathrm{mg}$ daily was added. 5-HTP was started at $50 \mathrm{mg}$ and titrated to $200 \mathrm{mg}$, the patient had normal sleep duration and continuity, and at $250 \mathrm{mg}$, he reported improved mood and continued relief of suicidal ideation. Mood remained euthymic for 4 months with titration of 5-HTP to $400 \mathrm{mg}$ orally twice daily. Nausea responded to promethazine. Eight months status-post sapropterin treatment, CSF neopterin and biopterin were in the normal range ( 10 and $12 \mathrm{nM} / \mathrm{l}$, respectively). The patient continues to report only minimal residual symptoms and improved function presently. To our
MAOI monoamine oxidase inhibitor, ECT electroconvulsive therapy, 5HTP 5-hydroxytryptophan, 5HIAA 5-hydroxyindoleacetic acid, HVA homovanillic acid, $\mathrm{BH} 4$ tetrahydrobiopterin (Color figure online)

knowledge, this is the first report of a severe biopterin metabolic defect and a pattern of metabolites consistent with GTPCH deficiency presenting with isolated severe psychiatric symptoms in the absence of neurologic abnormality. The etiology of the patient's severe CNS biopterin deficiency remains unclear, as GTPCH molecular studies were normal. However, this enzyme requires an additional protein subunit for activation, a defect that would reduce GTPCH activity. Previously unrecognized negative regulatory mechanisms on the enzyme may also be at play and could be identified through additional genome sequencing studies. The metabolic profile reported here is unique to GTPCH deficiency or a defect in the GTP activator protein with other enzymatic defects in the pterin synthetic pathway showing with very different metabolic patterns. It should be noted that little is known about alterations or responses in this pathway with neuroactive metabolic agents. For example, there could be non-specific secondary changes mimicking deficiencies that are not clinically relevant. Alterations in the pathway could be related to a pathophysiologic process or even pharmacotherapy, emphasizing the need for additional metabolomics 
studies. While it is possible that the discovery of a defect in the pterin biosynthetic pathway is coincidental to symptoms of severe, treatment-resistant depression and suicidality, the patient's clinical response to replacement of $\mathrm{BH} 4$ with 5-HTP supplementation makes this unlikely. Successful reversal of life-threatening depression refractory to known interventions does suggest that metabolic abnormalities should be considered in similar cases.

A second case of a more common metabolic disorder, cerebral folate deficiency [5-methyltetrahydrofolate (5MTHF) deficiency] presenting with primarily psychiatric symptoms has also identified by our team. Cerebral folate deficiency has been described as a CNS specific syndrome with low 5-MTHF in CSF and normal folate in plasma [28]. Folate is involved in nearly 100 metabolic reactions [29], and because folate is involved in the purine synthetic pathway, there may be an association between low 5-MTHF and impaired BH4 synthesis leading to impairment of serotonin, norepinephrine, and dopamine synthesis [28] (Fig. 1). In addition to its contribution to synthesis of monoamines implicated in psychiatric disorders, screening for low CSF 5-MTHF levels has been recommended in neurologic disorders of unknown origin [30,31].

Definitive diagnosis of cerebral folate deficiency requires CSF sampling. Our patient was a young woman with a significant major depressive disorder and sudden mood fluctuations. She had history of non-suicidal self-injury and five suicide attempts. Her symptoms partially remitted with high dose serotonin reuptake inhibitor and cognitive behavioral therapy, but she continued to have sudden dips in mood with significant suicidal ideation and recurrence of non-suicidal self injury despite treatment. CSF testing revealed low 5MTHF level of 35 (normal range 40-120). CSF 5-HIAA, HVA, neopterin, and biopterin were within normal limits. Plasma testing revealed normal hemoglobin (13.8), hematocrit (40), serum B12 (937), and folate (14.4) levels. Serine, glycine, and homocysteine levels were within normal limits. In light of her low CSF 5-MTHF, we initiated treatment with folinic acid [29] at $5 \mathrm{mg}$ PO daily. Folinic acid bypasses deconjugation and reduction steps for folic acid metabolism, i.e., metabolism occurs without dihydrofolate reductase. The patient reported reduction in mood fluctuation, resolution of suicidal ideation, and improvement in mood after 8 weeks. She denied side effects. Folinic Acid was increased to $10 \mathrm{mg}$ po daily at 8 weeks. In the following months, the patient reported continued stable improvement of mood.

\section{Conclusion}

A diagnosis of an inborn error of metabolism is often made in the setting of a family history of a known disorder, symptoms that are exacerbated by significant physiologic changes (such as fever, fasting, surgeries, or extremes of temperature), and otherwise unexplained multi-system disease. However, it is also important to consider isolated psychiatric symptoms as a sign of an IEM at times of stress or physiologic change such as puberty, a time of onset for many IEMs associated with psychiatric symptoms [1], childbirth (such as ornithine transcarbamylase disorder [32], and urea cycle disorders [33], and in times of physical trauma. In addition, expansion to other clinical situations is necessary, especially in the setting of a treatment resistant psychiatric disorder. As demonstrated by our patients, it is possible that adolescent and adult onset milder variants of inborn metabolic disease can escape diagnosis for many years, leading to ineffective or inadequate management of symptoms. Identification of a treatable inborn error of metabolism early in the course of psychiatric illness may reduce emotional stress, cognitive impairment, and developmental delay. At the very least, it can reduce stigma, and prevent adverse effects of ineffective treatments. Identification of new IEMs leading to psychiatric illness ultimately may allow us to repurpose currently approved standard and orphan drugs. Finally, with the identification of sentinel cases of IEMs contributing to psychiatric illness, we may be able to develop new peripheral screening tests for patients with severe or atypical psychiatric symptoms [34].

Disclosure L Pan: Career Development Award grant from NIMH/ NICHD K23 and BBFR/NARSAD grant; J Vockley: none.

\section{References}

Papers of particular interest, have been highlighted as:

- Of importance;

•- Of major importance

1. Sedel F, Baumann N, Turpin JC, et al. Psychiatric manifestations revealing inborn errors of metabolism in adolescents and adults. JIMD. 2007;30:631-41.

2. Trefz F, Maillot F, Motzfeldt K, Schwarz M. Adult phenylketonuria outcome and management. Mol Genet Metab. 2011;104(Suppl):26-30.

3. Waisbren S, White DA. Screening for cognitive and socialemotional problems in individuals with PKU: tools for use in the metabolic clinic. Mol Genet Metab. 2010;99(Suppl):96-9.

4. Shellmer DA, De Vito Dabbs A, Dew MA, Noll RB, et al. Cognitive and adaptive functioning after liver transplantation for maple syrup urine disease: a case series. Pediatr Transpl. 2011;15:58-64.

5. Walsh KS, Scott MN. Neurocognitive profile in a case of maple syrup urine disease. Clin Neuropsychol. 2010;24:689-700.

6. Svetel M, Potrebić A, Pekmezović T, et al. Neuropsychiatric aspects of treated Wilson's disease. Parkinsonism Relat Disord. 2009;15:772-5.

7. Millward LM, Kelly P, King A, Peters TJ. Anxiety and depression in the acute porphyrias. J Inherit Metab Dis. 2005;28:1099-107. 
8. Swift RG, Sadler DB, Swift M. Psychiatric findings in Wolfram syndrome homozygotes. Lancet. 1990;336:667-9.

9. Abbott MH, Folstein SE, Abbey H, et al. Psychiatric manifestations of homocystinuria due to cystathionine B-synthase deficiency: prevalence, natural history, and relationship to neurologic impairment and vitamin B6-responsiveness. AJMG. 1987;36: 959-69.

10. Bottiglieri T, Laundy M, Crellin R, et al. Homocysteine, folate, methylation, and monoamine metabolism in depression. JNNP. 2000;69:228-32.

11. Udden J, Folia V, Petersson KM. The neuropharmacology of implicit learning. Curr Neuropharmacol. 2010;8:367-81.

12. Chamberlain SR, Muller U, Blackwell AD, et al. Neurochemical modulation of response inhibition and probabilistic learning in humans. Science. 2006;311:861-863.

13. Scriver CR, Waters PJ. Monogenic traits are not simple: lessons from phenylketonuria. Trends Genet. 1999;15:267-72.

14. • Vockley J. Metabolism as a complex genetic trait, a systems biology approach: implications for inborn errors of metabolism. J Inherit Metab Dis. 2008;31:619-629. This article reviews the phenomenon of synergistic heterozygosity.

15. Vockley J, Rinaldo MJ, Bennett MJ, et al. Synergistic heterozygosity: disease resulting from multiple partial defects in one or more metabolic pathways. Mol Genet Metab. 2000;71:10-8.

16. Martianov I, Ramadass A, Barros AS, et al. Repression of the human dihydrofolate reductase gene by a non-coding interfering transcript. Nature. 2007;445:666-70.

17. Blau N, Bonafe L, Thony B. Tetrahydrobioterin deficiencies without hyperphenylalanemia: diagnosis and genetics of DOPAresponsive dystonia and sepiapterin reductase deficiency. Mol Genet Metab. 2002;74:172-85.

18. Werner ER, Blau N, Thony B. Tetrahydrobiopterin: biochemistry and pathophysiology. Biochem J. 2011;438:397-414.

19. - Pan L, McKain BW, Madan-Khetarpal S, et al. GTP-cyclohydrolase deficiency responsive to sapropterin and 5-HTP supplementation: relief of treatment-refractory depression and suicidal behavior. BMJ case reports 2011. doi:10.1136/bcr.03.2011.3927. This article describes a new disorder of neurotransmitter metabolism contributing to severe depression.

20. Bottiglieri T, Hyland K, Laundy M, et al. Folate deficiency, biopterin, and monoamine metabolism in depression. Psychol Med. 1992;22:871-6.

21. Niederwieser A, Ponzone A, Curtius HC. Differential diagnosis of tetrahydrobiopterin deficiency. J Inherit Metab Dis. 1985;8: 34-8.
22. - Paige LA, Mitchell MW, Krishnan KRR, et al. A preliminary metabolomic analysis of older adults with and without depression. Int J Geriatr Psychiatry. 2007;22:418-423. This article describes alterations of lipid and neurotransmitter metabolism in the depressed state.

23. Lotrich FE. Depression symptoms, low-grade inflammatory activity, and new targets for clinical intervention. Biol Psychiatry. 2011;70:111-2.

24. •• Quinones MP, Kaddurah-Daouk R. Metabolomics tools for identifying biomarkers for neuropsychiatric diseases. Neurobiol Dis. 2009;35:165-176. This article describes metabolomic studies in central nervous system specific disorders.

25. Manji H, Kato T, DiProspero NA, et al. Impaired mitochondrial function in psychiatric disorders. Nat Rev Neurosci. 2012;13: 293-307.

26. Walsh T, McClellan JM, McCarthy SE, et al. Rare structural variants disrupt multiple genes in neurodevelopmental pathways in schizophrenia. Science. 2008;320:539-43.

27. Nelson MR, Wegmann D, Ehm MG, et al. An abundance of rare functional variants in 202 drug target genes sequenced in 14,002 people. Science. 2012;337:100-4.

28. Ramaekers VT, Hausler M, Opladen T, et al. Psychomotor retardation, spastic paraplegia, cerebellar ataxia, and dyskinesia associated with low 5-methyltetrahydrofolate in cerebrospinal fluid: a novel neurometabolic condition responding to folinic acid substitution. Neuropediatrics. 2002;33:301-8.

29. Ramaekers VT, Blau N. Cerebral folate deficiency. DMCN. 2004;12:843-51.

30. Gordon N. Cerebral folate deficiency. DMCN. 2009;51:180-2.

31. Mangold S, Blau N, Opladen T, et al. Cerebral folate deficiency: a neurometabolic syndrome? Mol Genet Metab. 2011;104: 369-72.

32. Peterson DE. Acute postpartum mental status change and coma caused by previously undiagnosed ornitihine transcarbamylase deficiency. Obstet Gynecol. 2003;102:1212-4.

33. Fassier T, Guffon N, Acquaviva C, et al. Misdiagnosed postpartum psychosis revealing a late-onset urea cycle disorder. Am J Psychiatry. 2011;168:576-80.

34. Naylor EW, Ennis D, Davidson GF, et al. Guanosine triphosphate cyclohydrolase I deficiency: early diagnosis but routine urine pteridine screening. Pediatrics. 1987;79:374-8.

35. Saudubray JM, Walter JH, Van der Berghe G. Inborn metabolic diseases: diagnosis and treatment. Berlin: Springer; 2012. 